\title{
INEQUALITIES FOR THE FUNDAMENTAL ROBIN EIGENVALUE FOR THE LAPLACIAN ON $N$-DIMENSIONAL RECTANGULAR PARALLELEPIPEDS
}

\author{
G. KEADY AND B. WiWATANAPATAPHEE
}

\begin{abstract}
Amongst $N$-dimenstional rectangular parallelepipeds (boxes) of a given volume, that which has the smallest fundamental Robin eigenvalue for the Laplacian is the $N$-cube. We give an elementary proof of this isoperimetric inequality based on the well-known formulae for the eigenvalues. Also treated are various related inequalities which are amenable to investigation using the formulae for the eigenvalues.
\end{abstract}

Mathematics subject classification (2010): 26A51, 26B25, 35J05, 35P15, 52A40, 90C25. Keywords and phrases: Laplacian eigenvalue, Robin boundary condition.

\section{REFERENCES}

[1] M. Abramowitz ANd I. A. STEgun (Eds.), Handbook of Mathematical Functions with Formulas, Graphs, and Mathematical Tables, National Bureau of Standards, Applied Mathematics Series 55, 9th printing, Washington, 1970.

[2] G. D. Anderson, M. K. Vamanamurthy, M. Vuorinen, On the generalized convexity and concavity, J. Math. Anal. Appl 335 (2007), 1294-1308.

[3] A. BARICZ, Geometrically concave univariate distributions, J. Math. Anal. Appl. 363, 1 (2010), 182 196.

[4] Barkat Ali Bhayo And Li Yin, On the generalized convexity and concavity, arXiv: 1411.6586, 2014, 1-6.

[5] C. Borell, Greenian potentials and concavity, Mathematische Annalen 272, 1 (1985), 155-160.

[6] D. BuCUR AND A. GIACOMINI The St Venant inequality for the Laplace operator with Robin boundary conditions, Milan J. Math. 83, 2 (2015), 327-343.

[7] E. E. BURNISTON AND C. E. SIEWERT, The use of Riemann problems in solving a class of transcendental equations, Proc. Camb. Phil. Soc. 73, 1 (1973), 111-118.

[8] A. Coles ANTI, Brunn-Minkowski inequalities for variational functionals and related problems, Advances in Mathematics 194, 1 (2005), 105-140.

[9] R. Courant And D. Hilbert Methods of Mathematical Physics Interscience, 1957.

[10] D. DANERS A Faber-Krahn inequality for Robin problems in any space dimension, Math. Ann. 335, 4 (2006), 767-785.

[11] P. FREITAS AND B. SiUdeja Bounds for the first Dirichlet eigenvalue for triangles and quadrilaterals, ESAIM: Control Optimisation and Calculus of Variations 16, 3 (2010), 648-676.

[12] D. S. GREBENKov AND B.-T. NGUYEN Geometrical Structure of Laplacian Eigenfunctions, SIAM Rev. 55, 4 (2013), 601-667.

[13] K. Gustafson And T. ABE The third boundary condition was it Robin's, Mathematical Intelligencer 20 (1998), 63-71.

[14] G. H. Hardy, J. E. Littlewood and G. Polya, Inequalities, Cambridge U. P., 1934.

[15] J. HERSCH, Constraintes rectilignes parallèles et valeurs propres de membranes vibrantes, Z. Angew. Math. Phys. 17 (1966), 457-460.

[16] J.-B. Hiriart-URruty And J.-E. MartineZ-LegaZ, New formulas for the Legendre-Fenchel transform, J. Math. Anal. Appl. 288 (2003), 544-555. 
[17] G. KeAdy And A. MCNABB, Functions with constant Laplacian satisfying homogeneous Robin boundary conditions, I.M.A. Jnl. of Applied Mathematics 50 (1993), 205-224.

[18] G. Keady And B. Wiwatanapataphee, Supplement: Some isoperimetric results concerning unidirectional flows in microchannels, arXiv: 1604.03394, 2016, 1-68.

[19] G. KEADY AND B. WiWATANAPATAPHEE, Inequalities for the fundamental Robin eigenvalue of the Laplacian for box-shaped domains, arXiv:1705.09147, 2017, 1-60.

[20] G. Keady, N. Khajohnsaksumeth And B. Wiwatanapataphee, On unidirectional flows in microchannels with slip at the boundary, Proceedings of The International Conference on Engineering and Applied Science (TICEAS), 2018.

[21] R. S. Laugesen, Z. C. PAN And S. S. Son, Neumann eigenvalue sums on triangles are (mostly) minimal for equilaterals, Mathematical Inequalities and Applications 15, 2 (2012), 381-394.

[22] R. S. LAUgeSEn AND B. A. SiUdeJA, Sums of Laplace eigenvalues, rotationally symmetric maximizers in the plane, Journal of Functional Analysis 260, 6 (2011), 1795-1823.

[23] R. S. LAUGESEN, Tight frames and rotations: sharp bounds on eigenvalues of the Laplacian, Proceedings of the AMSI International Conference on Harmonic Analysis and Applications (Macquarie University), 2011.

[24] R. S. Laugesen And B. A. Siudeja, Sums of Laplace eigenvalues rotations and tight frames in higher dimensions, J. Math. Phys 52, 9 (2011), 093703, 13 pp.

[25] R. S. Laugesen And B. A. Siudeja, Triangles and Other Special Domains, in Shape optimization and spectral theory, De Gruyter Open, 2016.

[26] P. O. Lindberg, Power convex functions, in Generalised Concavity in Optimisation and Economics (S. Schalble, Ed.), Academic Press NY, 1982, 153-163.

[27] Q. LUO, Z. WANG AND J. HAN, A Padé approximant approach to two kinds of transcendental equations with applications in physics, European Journal of Physics 36, 3 (2017), 12 pp.

[28] V. E. Markushin, R. Rosenfelder And A. W. Schreiber, The $W_{t}$ Transcendental Function and Quantum Mechanical Applications, Italian Physical Society 1 (2002), 75-94.

[29] A. McNabb And G. Keady, Diffusion and the torsion parameter, J. Australian Math. Soc. 35B (1994), 289-301.

[30] M. MRŠEvČ, Convexity of the inverse function, The teaching of mathematics XI, 1 (2008), 21-24.

[31] C. P. Niculescu, L.-E. Persson, Convex functions and their applications: a contemporary approach, Springer, 2004.

[32] G. Polya, Sur le rôle des domaines symétriques dans le calcul de certaines grandeurs physiques, C. R. Acad. Sci. Paris 235 (1952), 1079-1081.

[33] G. Polya And G. Szego, Isoperimetric Inequalities in Mathematical Physics Princeton University Press, Princeton, 1951.

[34] R.L. Schilling, R. Song, Z. VondraceK, Bernstein Functions, Theory and Applications, De Gruyter, Studies in Mathematics 37, Berlin, 2010.

[35] R. SPERB, Bounds for the first eigenvalue of the elastically supported membrane on convex domains, Z. angew. Math. Phys. 54 (2003), 879-902. 